Supplemental Information:

\title{
Fragmentation of Saturated Hydrocarbons Upon Atmospheric Pressure Chemical Ionization is Caused by Proton Transfer Reactions
}

Jeremy M. Manheim, ${ }^{1, \#}$ Jacob R. Milton, ${ }^{1, \#}$ Y. Zhang, ${ }^{1}$ and Hilkka I. Kenttämaa ${ }^{1, *}$

* Corresponding author

\# These authors contributed equally to this work

1 Department of Chemistry, Purdue University, 560 Oval Drive, West Lafayette, IN 47907, West Lafayette, IN

Corresponding Author Email: hilkka@purdue.edu 
Fig. S1 Mass spectra measured following reactions between neutral $n$-butylcyclohexane and fragment ions of isooctane.

Fig. S2 Mass spectra measured following reactions between neutral $n$-butylcyclohexane and $m / z 83$.

Fig. S3 Mass spectrum of neat n-butylcyclohexane compared to ion-molecule reactions conducted between neutral n-butylcyclohexane and $\mathrm{m} / \mathrm{z} 32$ in the ion trap. S5

Discussion of the presence of even-mass ions in the APCI mass spectrum of neat n-butylcyclohexane. S5-S6

Details on the design of experiments related to the stainless-steel ion source.

Fig. S4 Schematic and design of the stainless-steel ion source.

Fig. S5 Mass spectra measured for the same lubricant base oil in two different ion sources-a commercial APCI source and the stainless-steel ion source

Fig. S6 APCI mass spectra for nitrogen gas with no added analyte, with and without applied accelerating voltages.... S9

Discussion of experiments used to determine the identity of ions of $m / z 29$ S9-S10

Fig. S7 Flowchart describing ion-molecule reaction results used to determine the identity of ions of $\mathrm{m} / \mathrm{z} 29$ S11

Fig. S8 Ion-molecule reaction mass spectra used to determine the identity of ions of $\mathrm{m} / \mathrm{z} 29$. S12

Fig. S9 CAD mass spectra of ions of $m / z 94$ and 93 . $\mathrm{S} 13$

Fig. S10 Ion-molecule reaction mass spectra measured following reactions between neutral isooctane and $\mathrm{N}_{2} \mathrm{H}^{+}$or protonated formaldehyde.

Fig. S11 Ion-molecule reaction mass spectra measured following reactions between neutral hexane and $\mathrm{N}_{2} \mathrm{H}^{+}$or protonated formaldehyde. 
(+)APCI/N 2

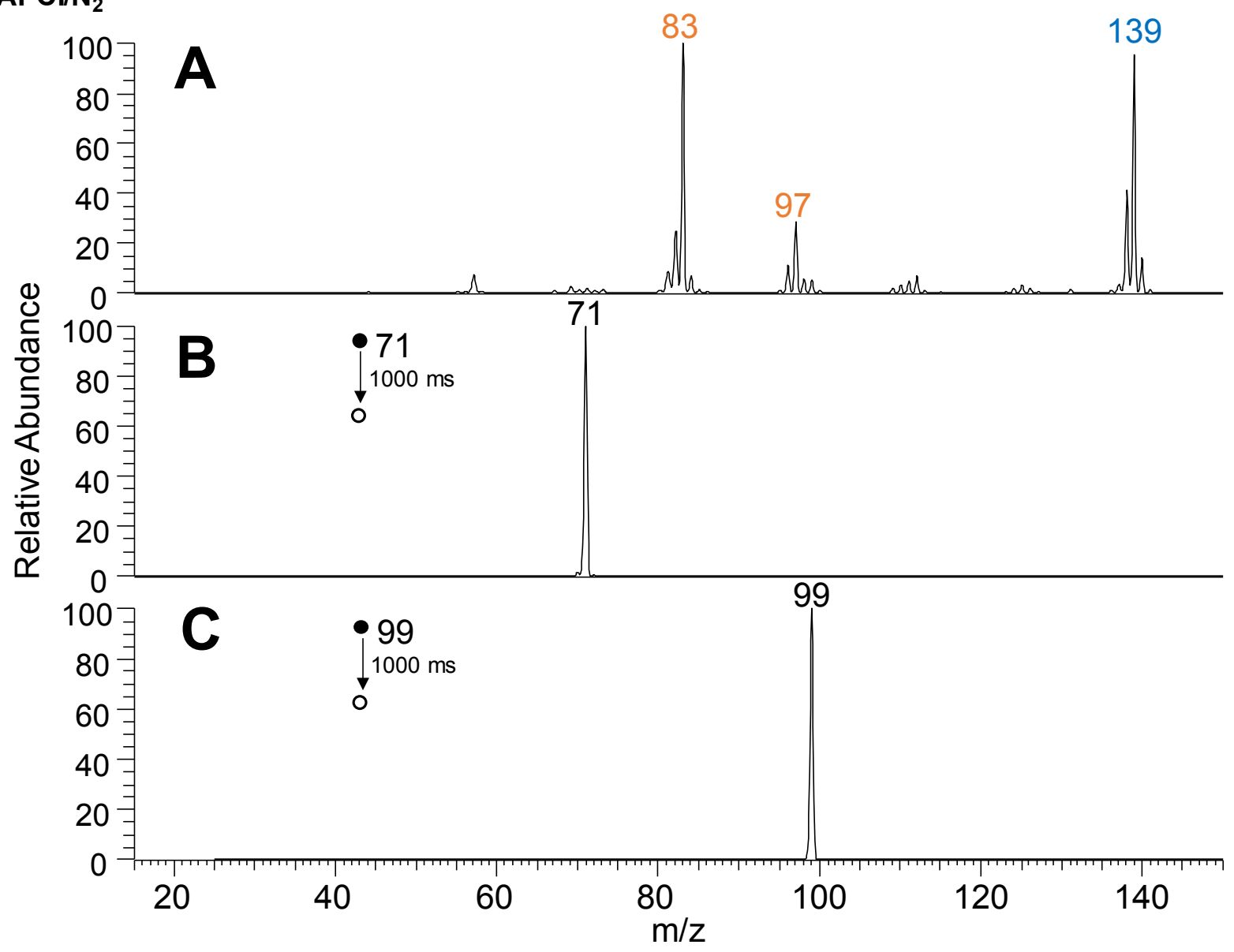

Figure S1. (A) (+)APCI mass spectrum of neat $n$-butylcyclohexane. The $m / z$-values of the $[\mathrm{M}-\mathrm{H}]^{+}$ion and other fragment ions of $n$-butylcyclohexane are colored blue and orange, respectively. (B) Mass spectrum measured following $1000 \mathrm{~ms}$ reactions of the fragment ions of $\mathrm{m} / \mathrm{z} 71$ and (C) $\mathrm{m} / \mathrm{z} 99$ of isooctane with $n$-butylcyclohexane in the ion trap. 
$(+) \mathrm{APCl} / \mathrm{N}_{2}$

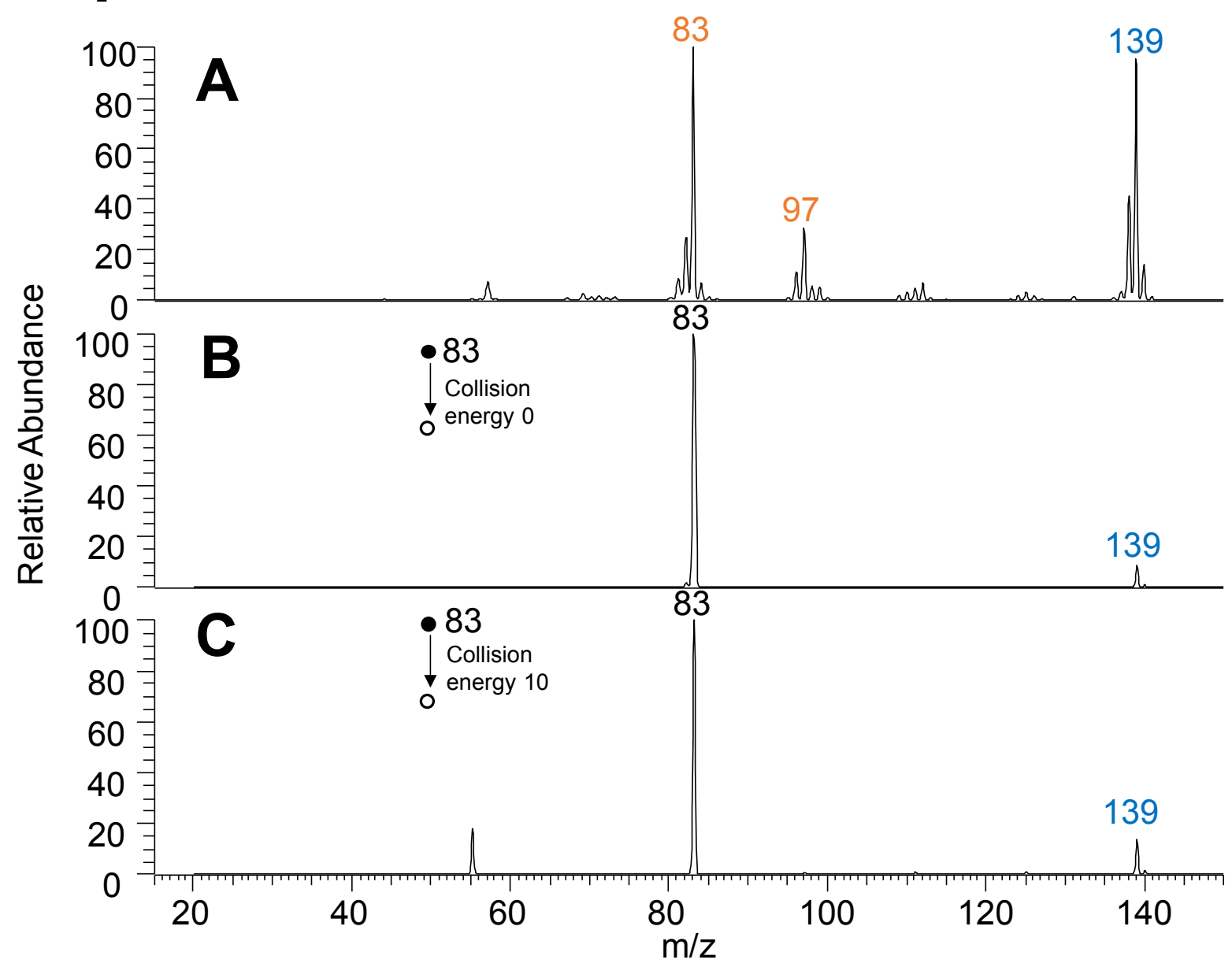

Figure S2. (A) (+)APCI mass spectrum of neat $n$-butylcyclohexane. The $m / z$-value of the $[\mathrm{M}-\mathrm{H}]^{+}$ions of $n$ butylcyclohexane is colored blue and those of the other fragment ions of $n$-butylcyclohexane are colored orange. Mass spectra measured in the ion trap following $500 \mathrm{~ms}$ reactions of the fragment ions of $\mathrm{m} / \mathrm{z} 83$ of $n$ butylcyclohexane with $n$-butylcyclohexane (B) without acceleration of the ions of $\mathrm{m} / \mathrm{z} 83$ and (C) at a normalized collision energy of 10 (arbitrary units). Can you add zoom-in into parts B and C? is the fragment ion 55 in C the same as seen in A at low mass? 


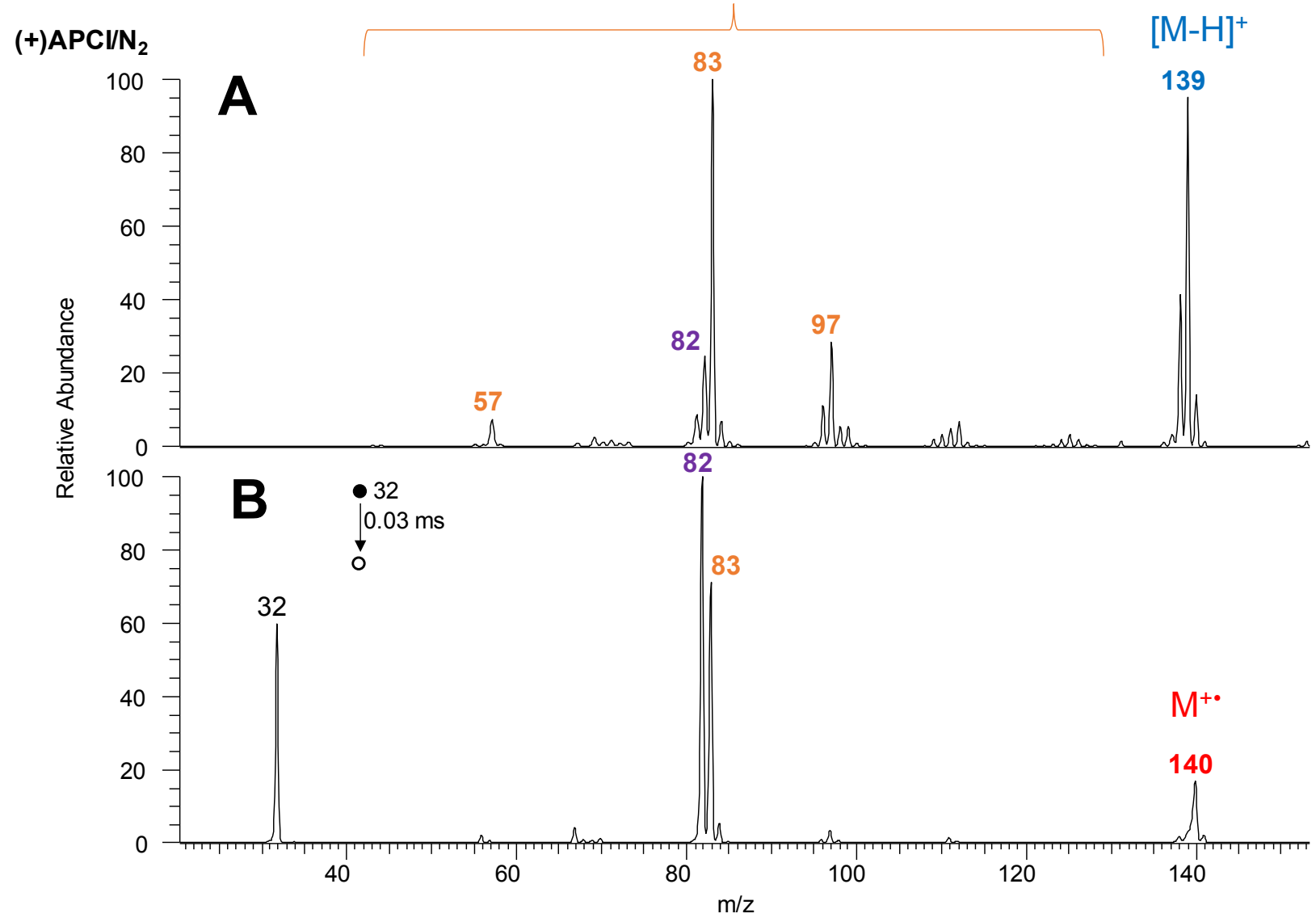

Figure S3. (A) (+)APCI mass spectrum of neat $n$-butylcyclohexane. (B) Mass spectrum measured following 0.03 ms reactions of isolated oxygen molecular ions of $m / z 32$ with $n$-butylcyclohexane in the ion trap. The $m / z$-values of the $[\mathrm{M}-\mathrm{H}]^{+}$ions of $n$-butylcyclohexane, the molecular ions of $n$-butylcyclohexane, the odd-mass fragment ions of $n$ butylcyclohexane, and the even-mass fragment ions of $n$-butylcyclohexane are colored blue, red, orange, and purple, respectively.

Even-mass Ions in APCI Spectra Reactions of even-mass ions, such as nitrogen-solvated nitrogen molecular ions and oxygen molecular ions, with neutral saturated hydrocarbons are likely to be the source of the few minor evenmass ions observed in the APCI mass spectrum of $n$-butylcyclohexane dissolved in isooctane (Figure 1A). In support of this hypothesis, even-mass fragment ions (i.e., radical cations) were only observed for reactions of oxygen molecular ions with $n$-butylcyclohexane; as discussed above, other reactions studied produced only oddmass fragment ions. This finding implies that while reactions between oxygen and nitrogen molecular ions and 
saturated hydrocarbons are not a major cause of fragmentation upon APCI, as these even-mass ions are present only in low abundancies in the APCI mass spectrum of $n$-butylcyclohexane (Figure 1B), such reactions are likely the reason that some even-mass ions can be observed.

Stainless steel ion source study. The stainless steel (SS) tube ion source design was first modeled using COMSOL Multiphysics and then built with the help of the Jonathan Amy facility in the Chemistry Department at Purdue University. A corona needle is situated inside of a SS tube with the point of the needle pointing approximately 16 $\mathrm{mm}$ from the exit of the SS tube ion source. A piece of peek is fixed between the stainless-steel tube and a metal insert to provide insulation. The inner diameter of the metal insert is $\sim 10 \mathrm{~mm}$. The corona needle, SS tube and insert each have their own voltages applied to them that can be controlled independently from one another. A stainlesssteel plate the size of the front cover of the Thermo Fisher Scientific API max house box was built to house the SS tube ion source (Figure S1; B). The exit of the SS tube ion source is $12 \mathrm{~mm}$ from the orifice of the ion transfer capillary tube of the mass spectrometer and is as close as possible to the nozzle of the commercial APCI source without blocking the nozzle opening (Figure S1; A). A syringe injection pump and several gas lines are connected externally to the SS tube ion source. Solvent and gas are mixed together and flow through the back of the SS tube ion source towards the corona needle where they are ionized. The voltages applied to the SS tube and insert act as focusing voltages that guide the ions to the MS inlet. As the reagent ions exit the SS tube they react with gaseous solvent and analyte introduced using a commercial APCI source without the corona discharge needle generating analyte ions, which enter the MS inlet.

The optimized parameters (parameters that produced the highest ion signal) were found to be $5 \mathrm{kV}, 3 \mathrm{kV}$, and $1.5 \mathrm{kV}$ on the corona needle, SS tube ion source, and the metal insert, respectively. The optimal solvent flow rate was found to be at $0.5 \mu \mathrm{L} / \mathrm{min}$ and oxygen flow rate was $3 \mathrm{~L} / \mathrm{min}$. Figure $\mathrm{S} 2$ compares the commercial APCI source and SS tube ion source mass spectra of a middle viscosity lubricant base oil dissolved in isooctane. Based on the work by Chunfen et al., ${ }^{16}$ the first distribution of ions represents the fragment ion region (and is denoted as such in Figure S5), and the second distribution of ions represents the $[\mathrm{M}-\mathrm{H}]^{+}$ions. The molecular weight distribution and ionization efficiency of the peaks observed in both mass spectra are similar. The ion signal for the middle viscosity sample when ionized using the SS tube ion source is an order of magnitude smaller than that of the commercial 
APCI source. The most likely reason for this is due to the distance of the corona needle from the ion source. The ions formed inside the SS tube must travel a much further distance to the ion transfer capillary than the ions formed in commercial APCI. In commercial APCI, the distance from the tip of the corona needle to the ion transfer capillary is $2 \mathrm{~mm}$ while in the SS tube design the distance is almost $28 \mathrm{~mm}$.

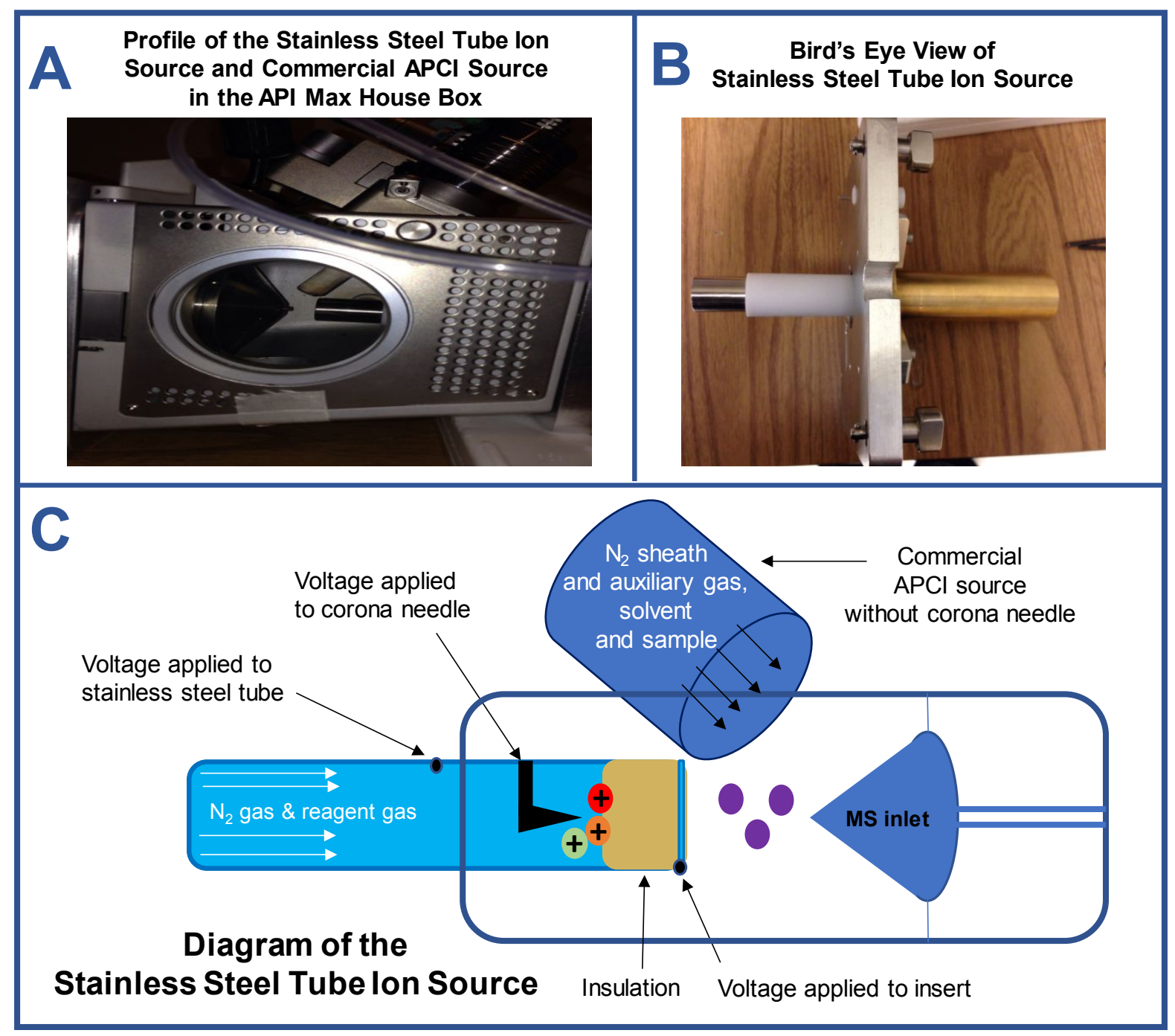

Figure S4. (A) Picture of the positioning of the stainless steel (SS) tube ion source in the API max house box. (B) Picture of the SS tube ion source. (C) Sketch of the SS tube ion source and commercial APCI source in the API house box. Voltages applied to the SS tube ion source and direction of sheath/auxiliary gas $\left(\mathrm{N}_{2}\right)$, reagent gas and sampleflow are indicated on the diagram. Red, orange, and green dots represent reagent ions generated in the SS 
tube ion source. Purple dots represent neutral analyte compounds that come from the APCI source without a corona discharge needle.

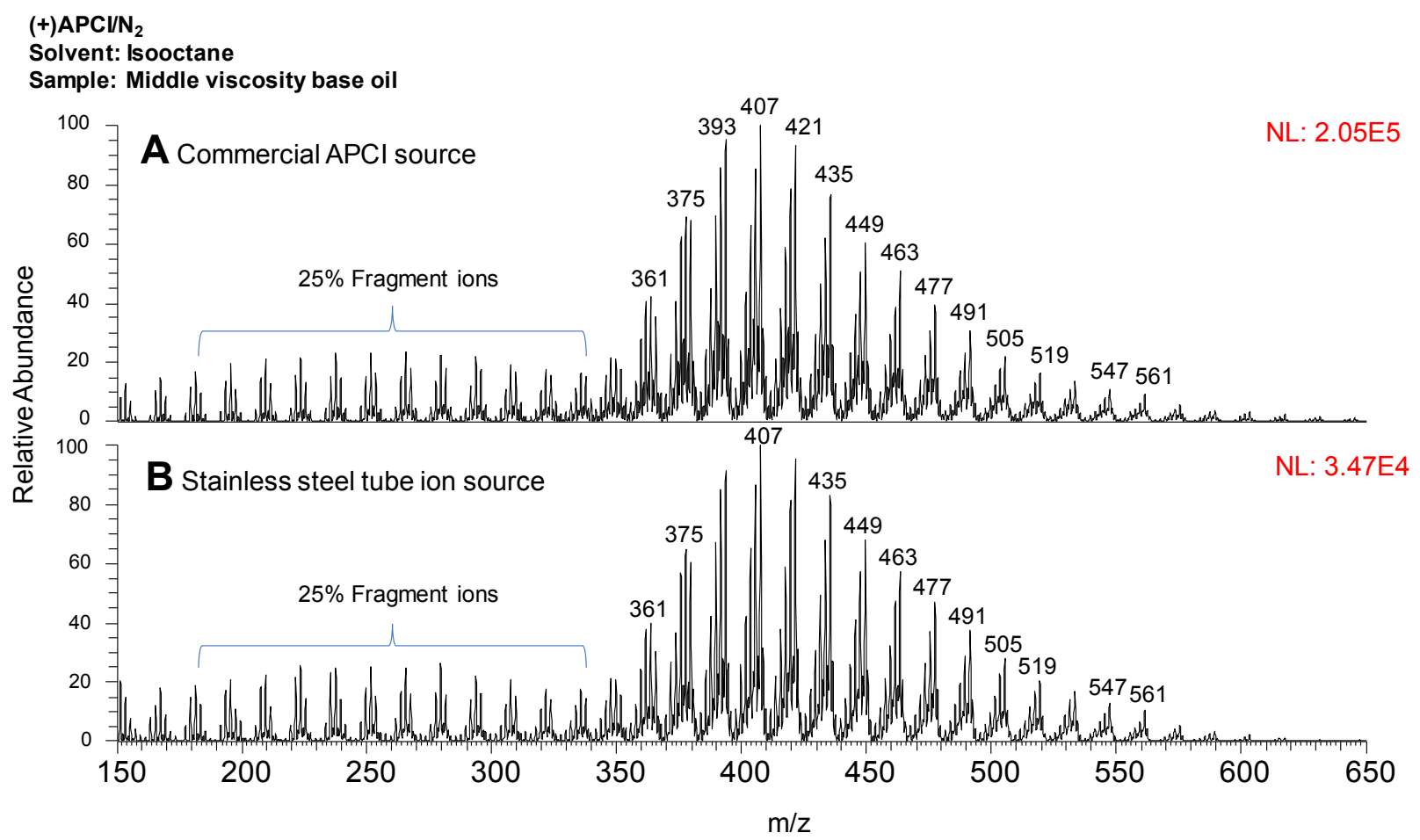

Figure S5. (A) (+)APCI mass spectrum measured for a base oil sample dissolved in isooctane by using a commercial APCI source. (B) (+)APCI mass spectrum measured for the same sample by using the stainless steel tube ion source. Based on the work by Chunfen at al., ${ }^{16}$ the first distribution of ions represents the fragment ion region (and is denoted as such in above Figure), and the second distribution of ions represents the $[\mathrm{M}-\mathrm{H}]^{+}$ions. 


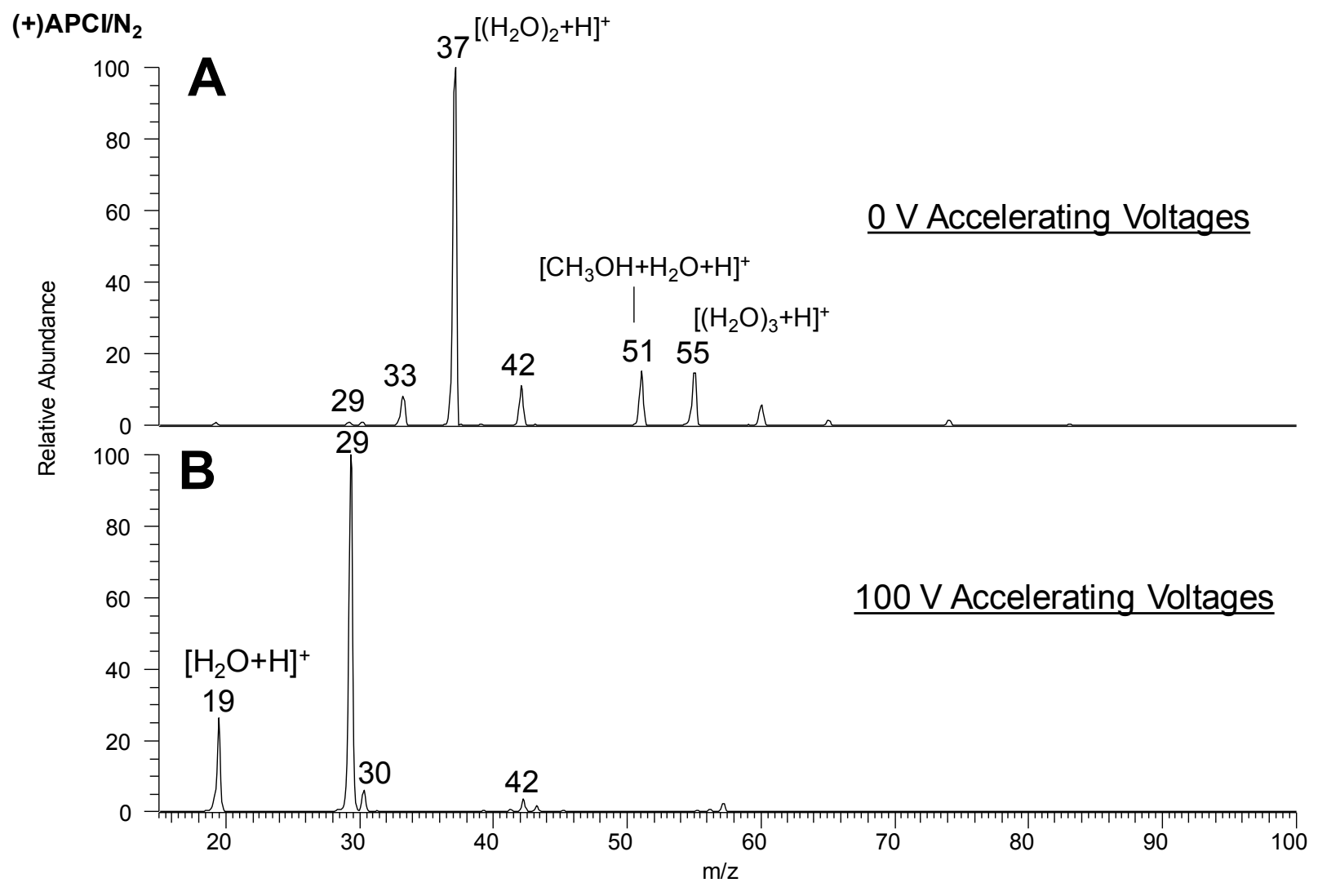

Figure S6. (A) (+)APCI mass spectrum measured for $\mathrm{N}_{2}$ gas with no accelerating voltages applied to the ion optics.

(B) (+)APCI mass spectrum measured for $\mathrm{N}_{2}$ gas with $100 \mathrm{~V}$ accelerating voltage applied to the ion optics.

Determining the identity of ion of $\mathbf{m} / \mathbf{z} \mathbf{2 9}$. The proton affinities of the four compounds that when protonated could form ions of m/z 29 are: $\mathrm{CH} 2 \mathrm{~N} \bullet, \mathrm{C} 2 \mathrm{H} 4, \mathrm{CO}$, and N2. Proton affinities (PA) for $\mathrm{C} 2 \mathrm{H} 4, \mathrm{CO}$, and $\mathrm{N} 2$ were all found on the NIST website. The PA of CH2N• was calculated at the B3LYP/6-311++G(d,p) level of theory. The protonation of each of these compounds is accompanied by their respective proton affinities in the equations below. The PA of benzene, $179.3 \mathrm{kcal} / \mathrm{mol}, 2,2,2$-trifluoroethanol, $167.4 \mathrm{kcal} / \mathrm{mol}$, hexafluorobenzene, $154.9 \mathrm{kcal} / \mathrm{mol}$, and $\mathrm{CO} 2,129.1 \mathrm{kcal} / \mathrm{mol}$, were all found on the NIST website and shown below. Ion-molecule reactions with benzene, 2,2,2-trifluoroethanol, and hexafluorobenzene were conducted by introducing each compound into the homebuilt manifold. Ion-molecule reactions were conducted with $\mathrm{CO} 2$ by replacing the helium collision gas that flows into the ion trap with $\mathrm{CO} 2$ gas. If a proton transfer reaction is observed between ions of $\mathrm{m} / \mathrm{z} 29$ and a neutral molecule, this implies the PA of the neutral molecule is greater than that of the ions of $m / z 29$. The example below describes the 
implication of observing, or not observing, protonated hexafluorobenzene in the ion-molecule reaction mass spectrum of ions of $\mathrm{m} / \mathrm{z} 29$ with neutral hexafluorobenzene.

Based on the composition of the atmosphere ( $78 \%$ nitrogen, $21 \%$ oxygen, $0.9 \%$ argon, and trace amounts of carbon dioxide, nitrous oxide, and water),34 the National Institute of Standards and Technology database was used to find previously observed ions of m/z 29 containing carbon, nitrogen, oxygen, and/or hydrogen.35 This led to four possible identities for the ions of $\mathrm{m} / \mathrm{z} 29$ : $\mathrm{CH} 2 \mathrm{NH}+\bullet, \mathrm{C} 2 \mathrm{H} 5+, \mathrm{HCO}+$, and $\mathrm{N} 2 \mathrm{H}+$. The conjugate base of each of these ions has a different proton affinity (PA 172, 162.6, 139.6, and $118.3 \mathrm{kcal}$ mol-1, respectively),35 and thus, by studying deprotonation reactions between the unknown ions of $\mathrm{m} / \mathrm{z} 29$ and several neutral reference bases with different known proton affinities, the identity of the ions of $\mathrm{m} / \mathrm{z} 29$ generated in the APCI experiments can be determined (see Figure S7). Figure S8 displays mass spectra measured after reactions of the unknown ions of m/z 29 with benzene (PA 179.3 kcalmol-1,35 top), hexafluorobenzene (PA 154.9 kcal mol-1,35 center) and carbon dioxide $(\mathrm{PA}=129.1 \mathrm{kcal}$ mol-1,35 bottom) in the ion trap. Generation of protonated benzene (Figure S8; top, m/z 79), protonated hexafluorobenzene (Figure S8; middle, m/z 187), and protonated carbon dioxide (Figure S8; bottom, m/z 45) was observed. The only structure proposed for the unknown ions of $\mathrm{m} / \mathrm{z} 29$ that is acidic enough (PA $118.1 \mathrm{kcal}$ mol-1)35 to protonate all these three reference bases is $\mathrm{N} 2 \mathrm{H}+$.

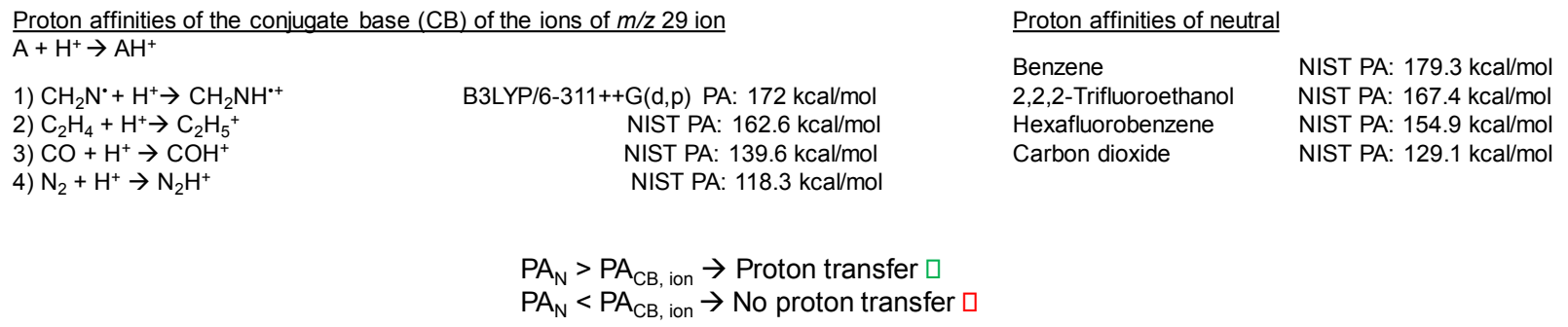

\footnotetext{
Example: if $\mathrm{PA}_{\text {hexafluorobenzene }}>\mathrm{PA}_{m / z} 29$, then ions of $m / z 29$ are either $\mathrm{COH}^{+}$ or $\mathrm{N}_{2} \mathrm{H}^{+}$; if, $\mathrm{PA}_{\text {hexafluorobenzene }}<\mathrm{PA}_{m / z} 29$, then ions of $m / z 29$ are either $\mathrm{CH}_{2} \mathrm{NH}^{++}$or $\mathrm{C}_{2} \mathrm{H}_{5}{ }^{+}$.
} 


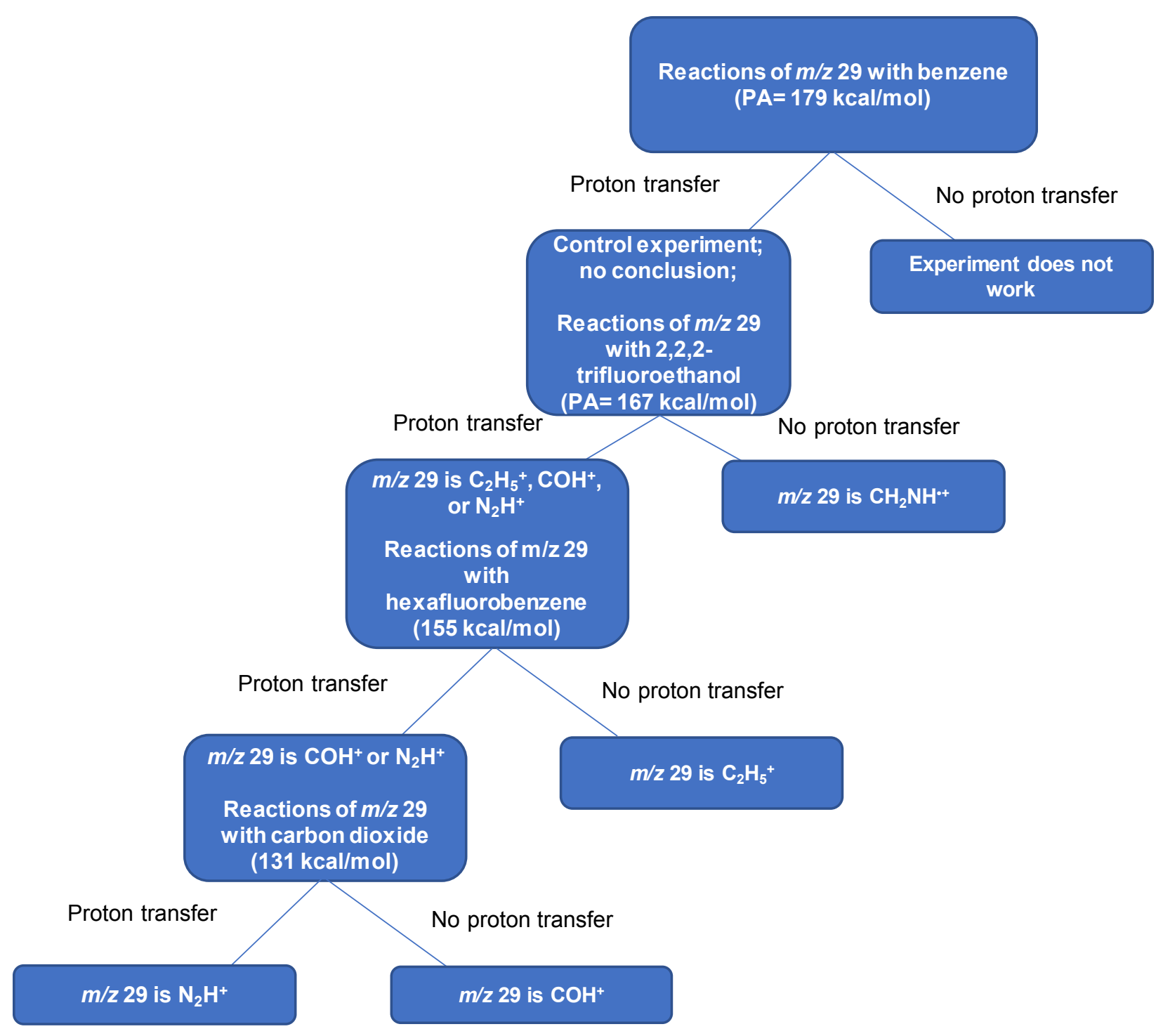

Figure S7. Flow chart representing the possible identities of ions of $\mathrm{m} / \mathrm{z} 29$ based on the results of protonation reactions 


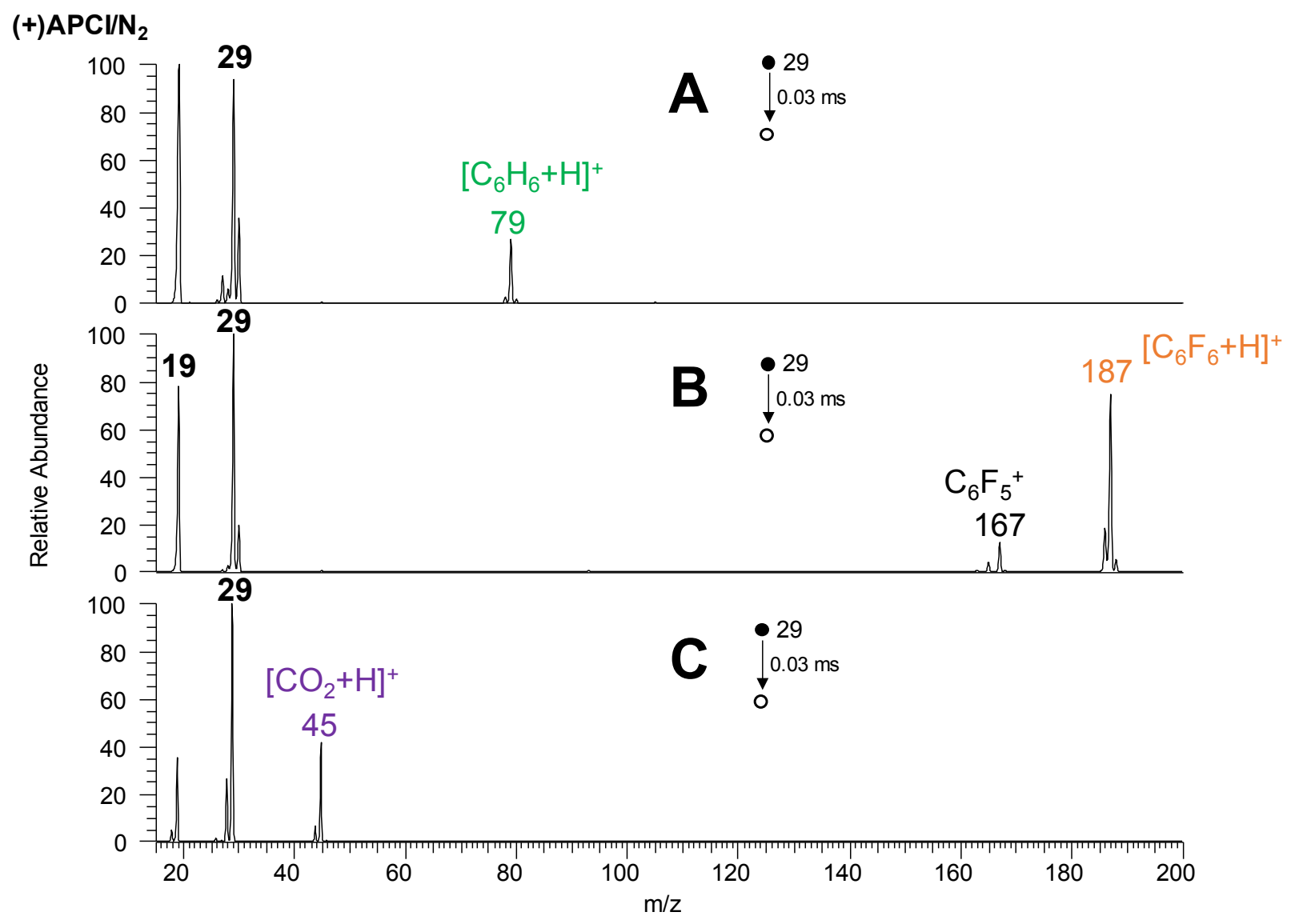

Figure S8. Mass spectra measured following $0.03 \mathrm{~ms}$ reactions of unknown ions of m/z 29 with (A) benzene (PA $179.3 \mathrm{kcal} / \mathrm{mol}$ ), (B) hexafluorobenzene (PA $154.9 \mathrm{kcal} / \mathrm{mol}$ ), and (C) carbon dioxide (PA $129.1 \mathrm{kcal} / \mathrm{mol}$ ) in the ion trap. The proton affinity of nitrogen is $118 \mathrm{kcal} / \mathrm{mol} .^{35}$ 
$(+) A P C I / N_{2}$

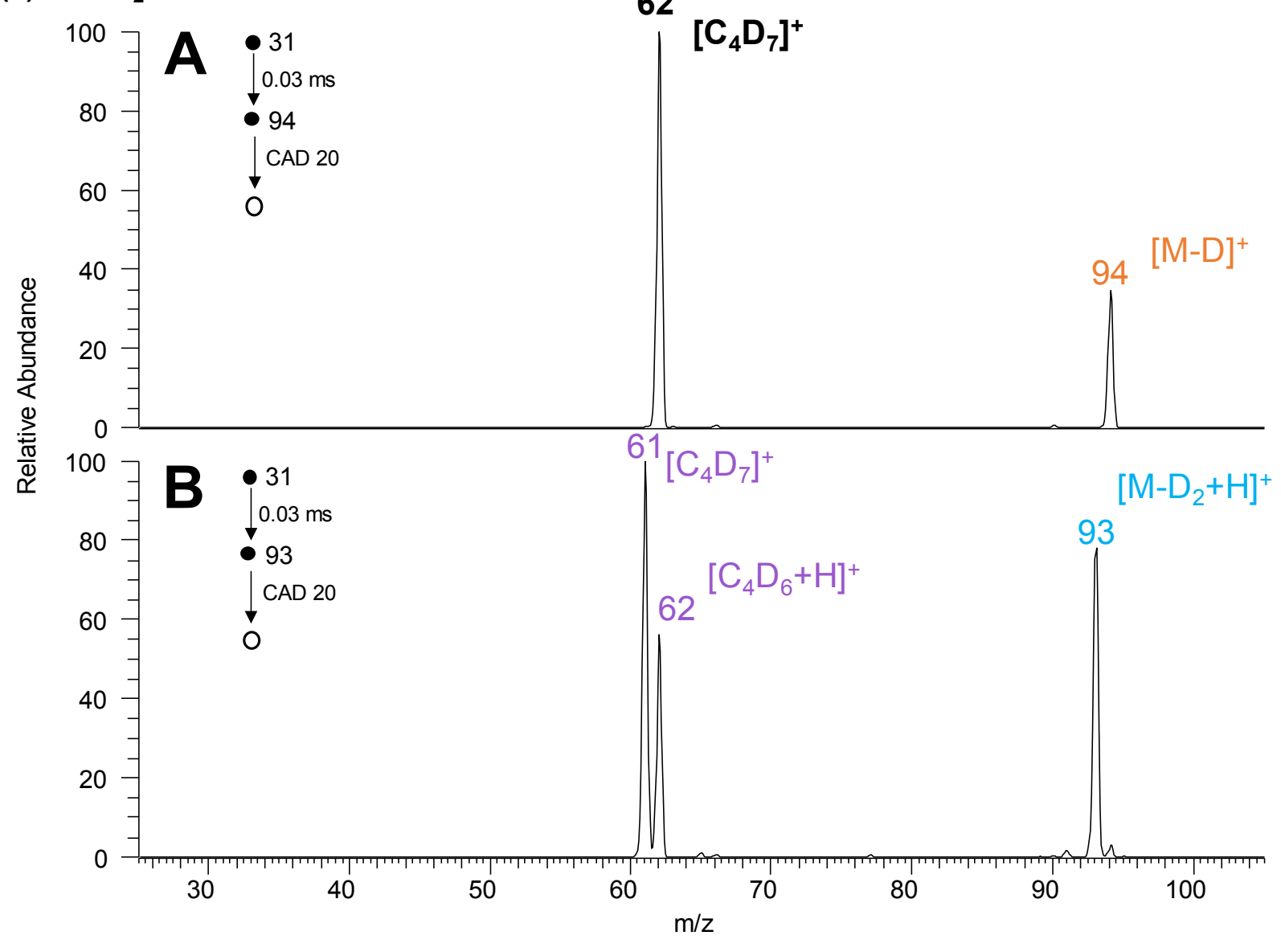

Figure S9. A CAD mass spectrum measured for the ions of $m / z 94$ ([M-D $]^{+}$; top) and the ions of $\mathrm{m} / z$ 93 ([M$\left.\mathrm{D}_{2}+\mathrm{H}\right]^{+}$; bottom) generated upon the reactions of protonated formaldehyde with cyclohexane- $\mathrm{d}_{12}$ in the ion trap. Mass spectra were measured at normalized collision energies of 20 . The $m / z$-value of the $[\mathrm{M}-\mathrm{D}]^{+}$ions of cyclohexane- $\mathrm{d}_{12}$ is colored orange, that of the fragment ions of $m / z$ is colored black, that of the $\left[\mathrm{M}-\mathrm{D}_{2}+\mathrm{H}\right]^{+}$ions of cyclohexane- $\mathrm{d}_{12}$ is colored blue, and that of the fragment ions of $\mathrm{m} / \mathrm{z} 93$ are colored purple. 


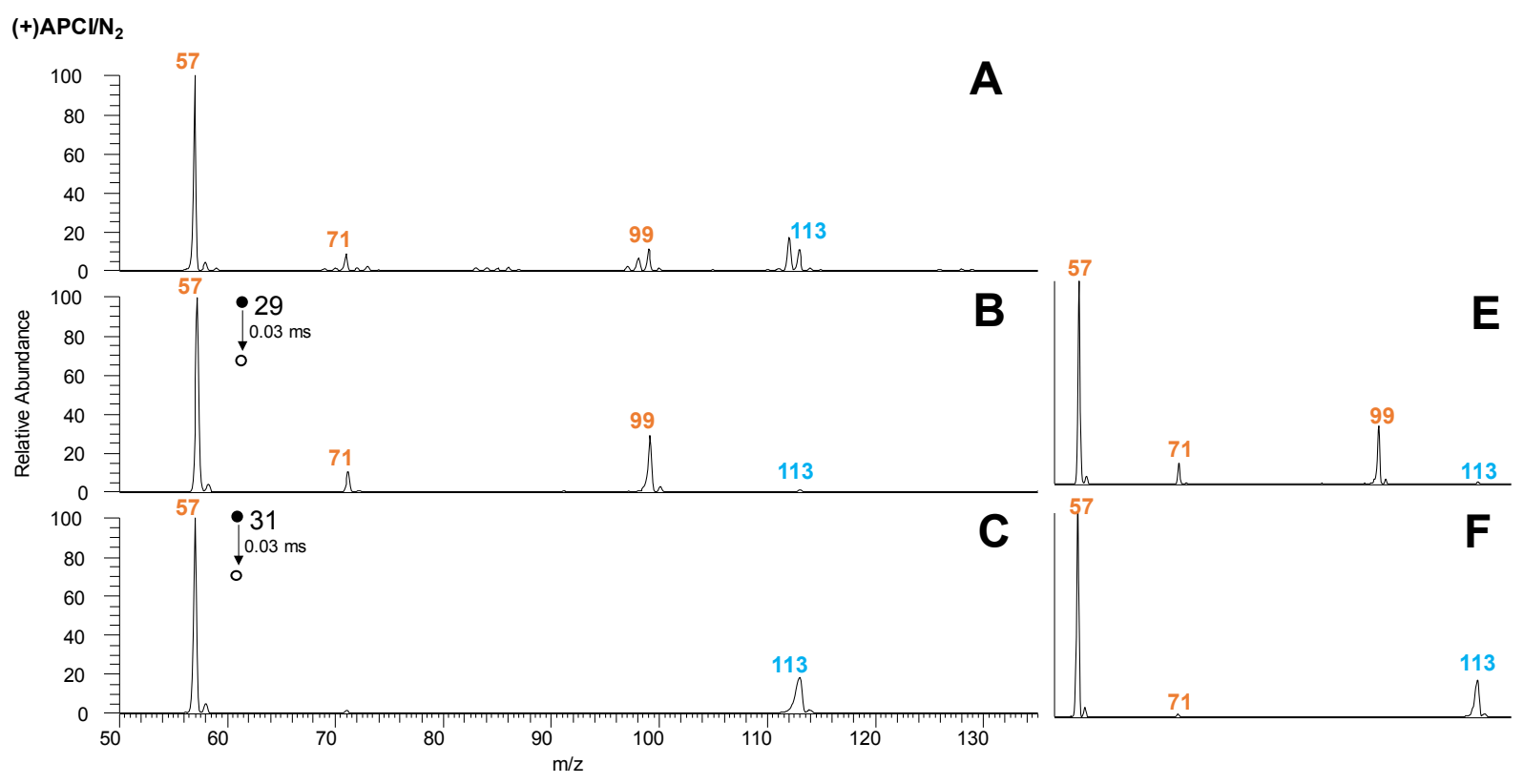

Figure S10. (+)APCI mass spectrum of neat isooctane (A). Full and zoomed-in mass spectra measured following $0.03 \mathrm{~ms}$ reactions of $\mathrm{N}_{2} \mathrm{H}^{+}(\mathrm{m} / z$ 29) with isooctane (B and E, respectively) in the ion trap. Full and zoomed-in mass spectra measured following $0.03 \mathrm{~ms}$ reactions of protonated formaldehyde $(\mathrm{m} / \mathrm{z} 31)$ with isooctane $(\mathrm{C}$ and $\mathrm{F}$, respectively) in the ion trap. The $m / z$-value of the $[\mathrm{M}-\mathrm{H}]^{+}$ion of isooctane is colored blue, that of the reactant ions are black, and those of the fragment ions are colored orange. 


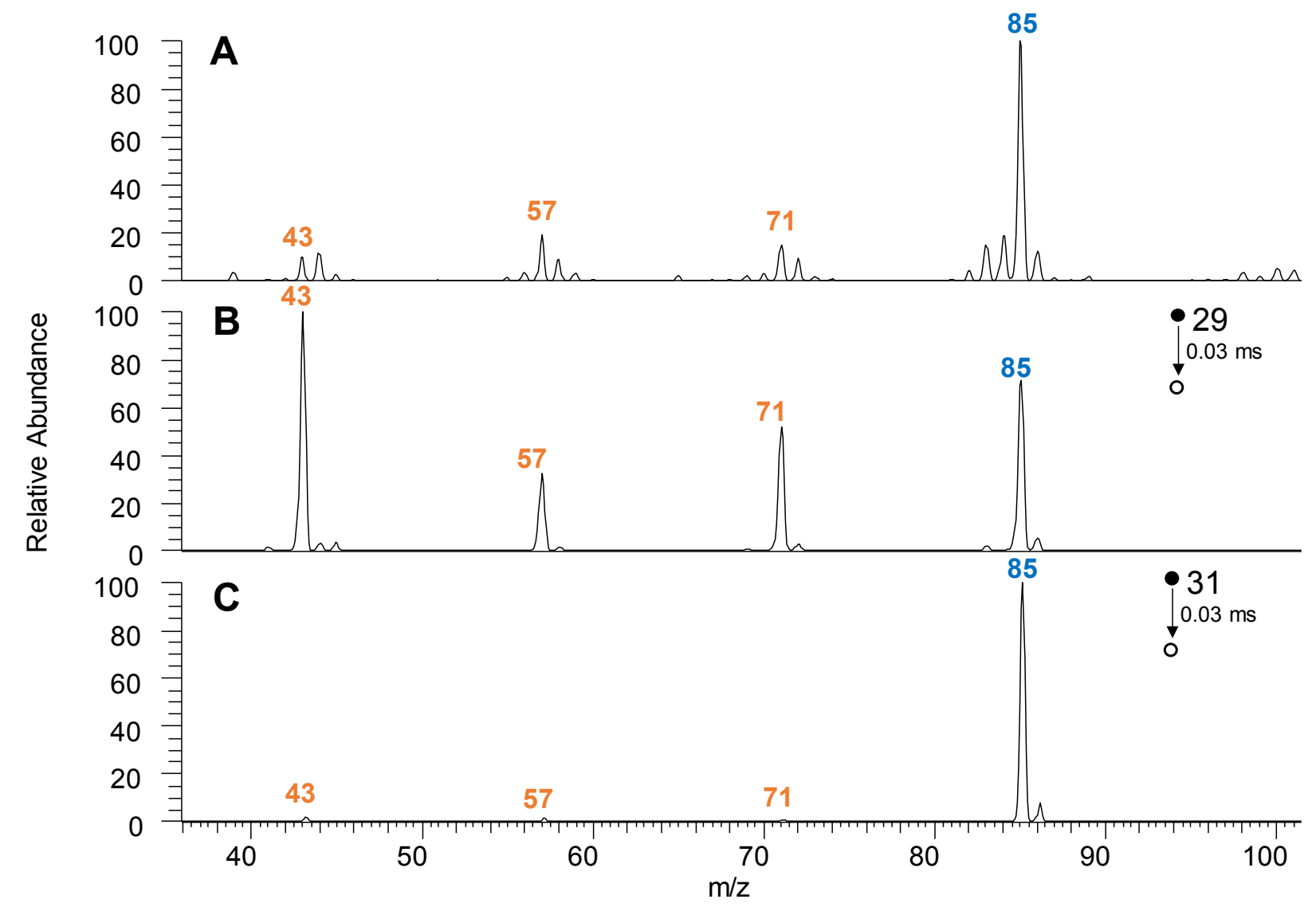

Figure S11. (+)APCI mass spectrum of neat n-hexane (A). A mass spectrum measured following 0.03 ms reactions of $\mathrm{N}_{2} \mathrm{H}^{+}(m / z 29)$ with n-hexane (B) in the ion trap. A mass spectrum measured following $0.03 \mathrm{~ms}$ reactions of protonated formaldehyde $(\mathrm{m} / \mathrm{z} 31)$ with n-hexane $(\mathrm{C})$ in the ion trap. The $m / z$-value of the $[\mathrm{M}-\mathrm{H}]^{+}$ions of n-hexane is colored blue and those of the fragment ions are orange. 